\title{
Uso do oxido nitroso como analgésico em parturientes: revisão integrativa
}

\author{
Use of nitrosous oxide as analgesic in parturientes: integrative review
}

Uso del oxido nitroso como analgésico en partículos: revisión integrativa

Kauan Gustavo de Carvalho ${ }^{1 *}$, Karla Joelma Bezerra Cunha ${ }^{2}$, Nanielle Silva Barbosa ${ }^{1}$, Joyce Carvalho Costa ${ }^{1}$, Ana Caroliny de Barros Soares Lima1', Lizandra Fernandes do Nascimento ${ }^{1}$, Ananda Carolina Barbosa da Silva ${ }^{1}$, Jessyca Fernanda Pereira Brito ${ }^{1}$, Larissa Vieira de Melo ${ }^{1}$, Ruth de Sousa Santos ${ }^{1}$, Viviane Gomes de Macedo ${ }^{1}$, Lívia Gabriela da Luz Carvalho', Maira Gislany de Castro Pereira ${ }^{1}$, Hortensia da Silva Lima Cruz ${ }^{1}$, Thalita Suellen Douglas Leone ${ }^{1}$.

\section{RESUMO}

Objetivo: Analisar evidências científicas relacionadas à utilização do oxido nitroso como analgésico em parturientes. Método: Estudo de revisão integrativa da literatura realizado nas bases de dados MEDLINE/Pubmed, CINAHL e LILACS utilizando-se os descritores: Analgesia, Trabalho de parto, Óxido nitroso e seus correspondentes em inglês. A coleta de dados ocorreu de novembro a dezembro de 2018. Resultados: Obteve-se como amostra sete artigos, apresentando, em sua maioria, nível de evidência 2B, colaborando para elaboração de duas categorias temáticas. Evidenciou-se que o óxido nitroso $\left(\mathrm{N}_{2} \mathrm{O}\right)$ é uma medida analgésica eficaz em parturientes, podendo ser utilizado em todos estados do parto, possuindo início de ação em segundos e eliminação rápida, sem comprometer feto e mãe. Quanto a assistência de enfermagem, cabe a coleta de informações, supervisão, avaliação clínica contínua e a administração à parturientes, exigindo uma supervisão e avaliação precisa da vitalidade fetal e bem-estar materno. Conclusão: A utilização do oxido nitroso é uma alternativa segura para o alívio da dor em parturientes. Pretende-se estimular e subsidiar novas pesquisas que trabalhem e aprofundem o uso do oxido nitroso além de outros métodos farmacológicos e não farmacológicos que possam ser aplicados para o alivio da dor durante o parto.

Palavras chave: Analgesia, Trabalho de parto, Óxido nitroso.

\begin{abstract}
Objective: To analyze scientific evidence related to the use of nitrous oxide as an analgesic in parturients. Method: An integrative review of the literature performed in the MEDLINE / Pubmed, CINAHL and LILACS databases using the descriptors: Analgesia, Labor, Nitrous Oxide and their correspondents in English. Data collection occurred from November to December 2018. Results: Obtained seven articles were sampled, mostly presenting evidence level $2 \mathrm{~B}$, collaborating to elaborate two thematic categories. It has been shown that nitrous oxide (N2O) is an effective analgesic measure in parturients, and can be used in all stages of labor, with onset of action in seconds and rapid elimination without compromising fetus and mother. Regarding nursing care, information collection, supervision, continuous clinical evaluation and administration to the parturients are required, requiring an accurate supervision and evaluation of fetal vitality and maternal well-being. Conclusion: The use of nitrous oxide is a safe alternative for the relief of pain in parturients. It is intended to stimulate and subsidize new researches that work and deepen the use of nitrous oxide in addition to other pharmacological and non-pharmacological methods that can be applied for the relief of pain during childbirth.
\end{abstract}

Key words: Analgesia, Labor Obstetric e Nitrous Oxide.

${ }^{1}$ Universidade Estadual do Piauí, Teresina, Piauí, Brasil. *E-mail: kauancarvalho2008@gmail.com

2 Universidade do Vale do Paraíba/Instituto de Pesquisa e Desenvolvimento, São José dos Campos, São Paulo, Brasil. 


\section{RESUMEN}

Objetivo: Analizar evidencias científicas relacionadas con la utilización del oxido nitroso como analgésico en parturientes. Método: Estudio de revisión integrativa de la literatura, realizado en las bases de datos, MEDLINE / Pubmed, CINAHL y LILACS, se utilizaron los descriptores: Analgesia, Trabajo de parto, Óxido nitroso y sus correspondientes en inglês. La recolección de datos ocurrió de noviembre a diciembre de 2018. Resultados: Se obtuvo como muestra siete artículos, presentando, en su mayoría, nivel de evidencia 2B, colaborando para elaboración de dos categorías temáticas. Se demostró que el óxido nitroso (N2O) es una medida analgésica eficaz en parturientes, pudiendo ser utilizado en todos los estados del parto, poseyendo inicio de acción en segundos y eliminación rápida, sin comprometer feto y madre. En cuanto a la asistencia de enfermería, cabe la recolección de informaciones, supervisión, evaluación clínica continua y la administración a parturientes, exigiendo una supervisión y evaluación precisa de la vitalidad fetal y bienestar materno. Conclusión: La utilización del oxido nitroso es una alternativa segura para el alivio del dolor en parturientas. Se pretende estimular y subsidiar nuevas investigaciones que trabajen y profundicen el uso del oxido nitroso además de otros métodos farmacológicos y no farmacológicos que puedan ser aplicados para el alivio del dolor durante el parto.

Palabras clave: Analgesia, Trabajo de parto, Óxido nitroso.

\section{INTRODUÇÃO}

O Oxido Nitroso caracteriza-se como um gás inodoro, insípido e antiinflamário, sintetizado pela primeira vez pelo cientista inglês Joseph Priestly em 1772 sendo utilizado pela primeira vez como analgésico durante o parto por Stanislav Klikovich, na Polônia, em 1881. Este gás pode ser aplicado para analgesia durante todas as fases do trabalho de parto, bem como nos procedimentos pós-parto, como na correção de laceração, remoção manual da placenta e/ou curetagem uterina (COLLINS M, et al., 2015).

Relatórios elaborados recentemente defendem a ampliação do acesso às opções seguras e menos invasivas que promovam conforto e alivio à dor no trabalho de parto, a exemplo, um programa desenvolvido na Universidade da Califórnia, em São Francisco (UCSF), que tem oferecido oxido nitroso como analgésico durante às parturientes por mais de 30 anos. Logo, é evidente que sua utilização é segura e tem sido discutida em países desenvolvidos por vários anos. Recentemente, o Centro Médico da Universidade de Vanderbilt (VUMC) iniciou com sucesso um programa ampliar o acesso a esse e outros tipo de analgesia nesse contexto (BOBB LE, et al., 2016).

Por ser tratar de um método não invasivo, o óxido nitroso oferece uma opção que agrada às mulheres que desejam analgesia no parto sem causar bloqueio neuroaxial conforme demonstrado em estudo realizado na França, em 2010, onde $26 \%$ das mulheres em estudo expressaram uma preferência por parto sem analgesia neuroaxial (SUTTON CD, et al., 2017).

A Sociedade Americana de Anestesiologia descreve o oxido nitroso como um tipo de analgesia com sedação mínima e efeitos adversos insignificantes. Essa categorização foi fundamental para estabelecer o papel dos enfermeiros na administração dessa modalidade terapêutica. Ainda de acordo com as essas diretrizes, uma mistura de $50 \%$ do oxido com $50 \%$ de oxigênio fornece efeitos sedativos e ansiolíticos mínimos fato que justificaria sua administração por enfermeiros obstetras às mulheres em trabalho de parto. Também é valido ressaltar que os enfermeiros fornecem cuidados contínuos diretos, e podem avaliar de forma longitudinal a eficácia dessa terapia (BARBIERI R, et al., 2014).

Entende-se que a composição farmacológica do óxido nitroso oferece riscos míninos a mãe e ao feto, portanto, esse se tornou uma alternativa apropriada para muitas mulheres.

Considerando a complexidade da terapêutica e manejo da dor que envolve o trabalho de parto, o presente estudo objetiva analisar, com base na literatura atual, as evidências científicas da ação analgésica do óxido nitroso durante o trabalho de parto, subsidiando o enriquecimento e expansão de pesquisas na área obstétrica. 


\section{METODOLOGIA}

Trata-se de revisão integrativa da literatura, fundamentada em referencial teórico, conduzido por seis etapas de investigação: identificação do tema e seleção da hipótese ou questão de pesquisa; estabelecimento de critérios de inclusão e exclusão de estudos/amostragem ou busca na literatura; definição das informações a serem extraídas dos artigos selecionados/categorização dos estudos; avaliação dos estudos incluídos na revisão integrativa; interpretação dos resultados e apresentação do revisão/síntese do conhecimento (MENDES KDS, et al., 2008).

Para elaboração da questão de pesquisa foi utilizado a estratégia PICO (Analgesia, Trabalho de parto, Óxido nitroso) (LOCKWOOD C, et al., 2017), resultando na seguinte questão: Quais as evidências científicas relacionadas a à utilização do oxido nitroso como analgésico em parturientes?

Foram considerados como critérios de inclusão: estudos primários disponíveis na íntegra nas bases de dados selecionadas. Excluiu-se estudos de abordagem qualitativas, revisões de literatura, duplicados, teses e dissertações.

A busca foi realizada por dois revisores, de forma simultânea e independente, no período de Novembro a Dezembro de 2018, nas bases de dados: Literatura Latino-Americana e do Caribe em Ciências da Saúde (LILACS), Medical Literature Analysis and Retrieval Sistem Online (MEDLINE/Pubmed) e Cummulative Index to Nursing and Allied Health Literature (CINAHL).

Utilizou-se os descritores indexados nos Descritores em Ciências da Saúde (DeCS) e no Medical Subject Headings (MeSH), combinados por operadores boleanos "AND" e "OR" gerando expressões de busca conforme Quadro 1.

Quadro 1 - Expressões de busca aplicadas às bases de dados conforme descritores selecionados mediante estratégia PICO. Teresina, PI, 2018.

\begin{tabular}{|c|c|c|c|}
\hline \multicolumn{4}{|c|}{ Descritores em Ciências da Saúde - DECS } \\
\hline \multicolumn{2}{|r|}{ DESCRITORES } & BASE DE DADOS & EXPRESSÃO FINAL DE BUSCA \\
\hline $\mathbf{P}$ & Analgesia & \multirow[t]{4}{*}{ LILACS } & \multirow{4}{*}{$\begin{array}{l}\text { Analgesia AND trabalho de parto AND } \\
\text { óxido nitroso }\end{array}$} \\
\hline I & Trabalho de parto & & \\
\hline C & \multirow[t]{2}{*}{ Óxido nitroso } & & \\
\hline 0 & & & \\
\hline \multicolumn{4}{|c|}{ Medical Subject Headings - MESHe/ list CINAHL } \\
\hline \multicolumn{2}{|r|}{ DESCRITORES } & BASE DE DADOS & EXPRESSÃO FINAL DE BUSCA \\
\hline $\mathbf{P}$ & Analgesia & MEDLINE/Pubmed & \multirow{4}{*}{$\begin{array}{l}\text { Analgesia AND labor obstetric AND } \\
\text { nitrous oxide }\end{array}$} \\
\hline I & Labor obstetric & \multirow[t]{3}{*}{ CINAHL } & \\
\hline C & Nitrous oxide & & \\
\hline 0 & & & \\
\hline
\end{tabular}

Fonte: Dados da pesquisa.

Para coleta de dados utilizou-se instrumento elaborado pelos autores deste estudo, contendo informações acerca do ano de publicação, país de origem, detalhamento do método e principais conclusões. Para a classificação do nível de evidência utilizou-se a recomendação do Oxford Centre for Evidence-based Medicine (DURIEUX N, et al., 2013).

No primeiro momento foi realizada a busca nas bases de dados e exclusão dos duplicados. Em seguida foi realizada revisão do título e resumo para inclusão dos estudos primários pertinentes. No momento seguinte, e de forma também independente, houve a leitura do texto completo dos artigos. As discordâncias foram resolvidas por meio de discussão com terceiro revisor (Figura 1). 
Figura 1 - Estabelecimento dos critérios de inclusão e exclusão dos artigos nas bases de dados LILACS, CINAHL e MEDLINE/Pubmed. Teresina, PI, 2018.

№ de relatos identificados nos bancos LILACS (1), CINAHL (10), MEDLINE/Pubmed (115)

$\mathrm{N}=126$

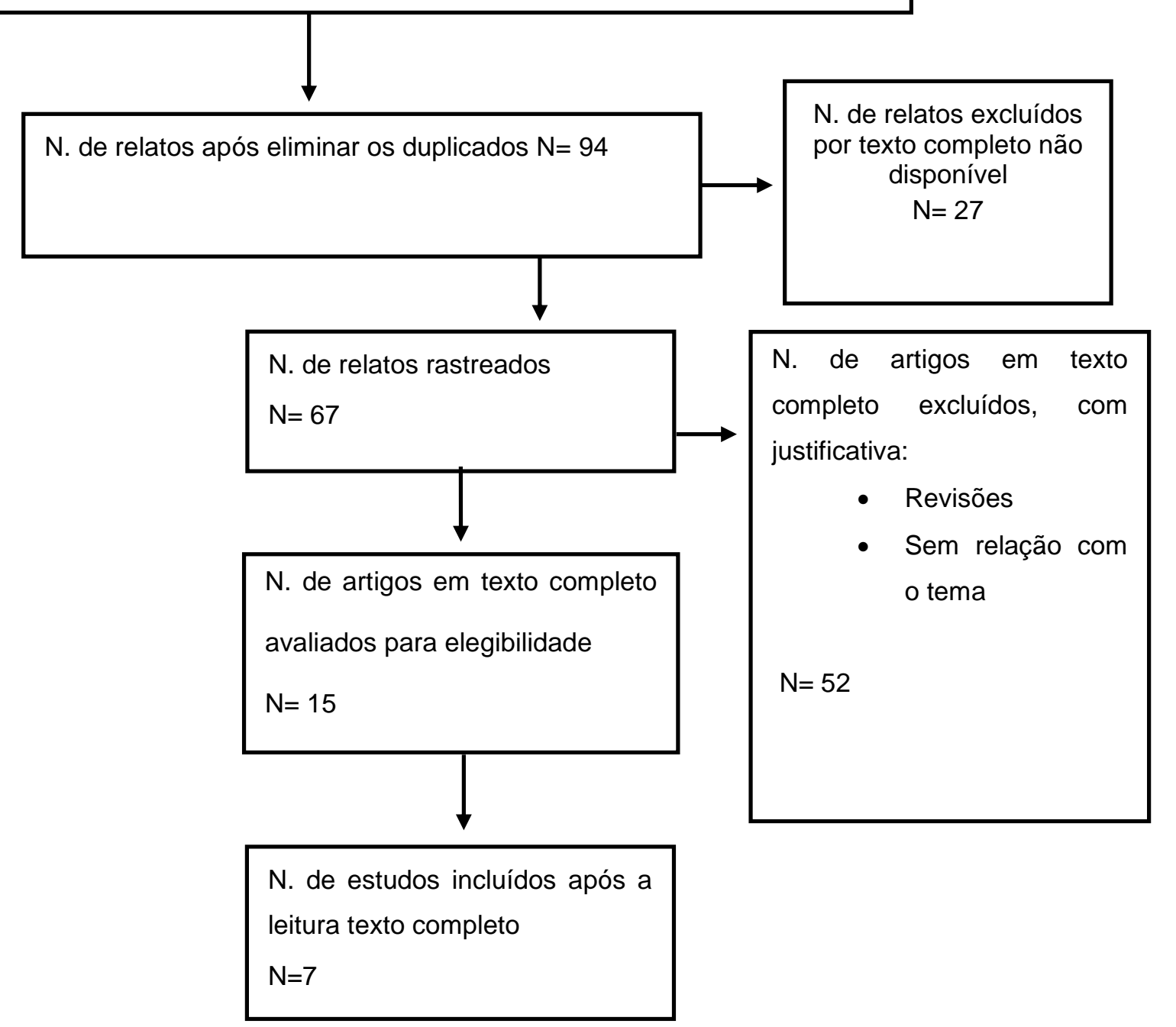

Fonte: Dados da pesquisa.

\section{RESULTADOS}

Os sete artigos selecionados foram pulicados no período entre 1994 a 2017. No ano de 2017 (MIGLIACCIO L, et al., 2017; RICHARDSON MG, et al., 2017) e 2012 (COLLINS M, et al., 2012; STEWART LS e COLLINS M, 2012) foram publicados dois artigos cada.

Quanto ao local de pesquisa, quatro artigos foram publicados na América do Norte (CARSTONIU J, et al., 1994; COLLINS M, et al., 2012; STEWART LS e COLLINS M, 2012; MIGLIACCIO L, et al., 2017), sendo o continente com maior número de pesquisa nesta temática. Ressalta-se que não foi encontrado nenhum artigo brasileiro em todas as etapas da pesquisa e que também não foi delimitado nenhum limite temporal (Quadro 2). 
Quadro 2 - Distribuição das referências por autor, ano de publicação, país de origem, área de atuação dos autores, nível de evidencia e delineamento do método. Teresina, PI, 2018.

\begin{tabular}{|c|c|c|c|c|}
\hline Autor/Ano & País & NE & Delineamento do método & Principais Resultados \\
\hline $\begin{array}{l}\text { BURGOS J., et } \\
\text { al. (2013) }\end{array}$ & Espanha & $2 \mathrm{~B}$ & $\begin{array}{l}\text { Tipo de estudo: Estudo de } \\
\text { coorte. } \\
\text { Amostra: } 300 \text { mulheres com } \\
\text { gestação única em apresentação } \\
\text { pélvica a termo }\end{array}$ & $\begin{array}{l}\text { Diminuiu o nível de dor } \\
\text { severa experimentada } \\
\text { pelas parturientes, sem } \\
\text { produzir prejuízos para } \\
\text { maternos e fetais. }\end{array}$ \\
\hline $\begin{array}{l}\text { CARSTONIU J, } \\
\text { et al. (1994) }\end{array}$ & Canadá & 1B & $\begin{array}{l}\text { Tipo de estudo: Estudo } \\
\text { randomizado controlado. } \\
\text { Amostra: } 29 \text { mulheres em } \\
\text { trabalho de parto. }\end{array}$ & $\begin{array}{l}\text { Os escores de dor não } \\
\text { diferiram } \\
\text { significativamente ao } \\
\text { longo do tempo em } \\
\text { função da substância } \\
\text { inalada, quando } \\
\text { comparado o óxido } \\
\text { nitroso com o ar } \\
\text { comprimido. }\end{array}$ \\
\hline $\begin{array}{l}\text { COLLINS M, et } \\
\text { al. (2012) }\end{array}$ & EUA & $2 \mathrm{~B}$ & $\begin{array}{l}\text { Tipo de estudo: Estudo de } \\
\text { coorte. } \\
\text { Amostra: } 25 \text { mulheres em } \\
\text { trabalho de parto. }\end{array}$ & $\begin{array}{l}\text { Analgesia do oxido } \\
\text { nitroso durante as } 3 \\
\text { fases sem efeitos } \\
\text { adversos ou prejuízos } \\
\text { fetais }\end{array}$ \\
\hline $\begin{array}{l}\text { MIGLIACCIO L, } \\
\text { et al. (2017) }\end{array}$ & México & $2 \mathrm{~B}$ & $\begin{array}{l}\text { Tipo de estudo: Estudo de } \\
\text { coorte. } \\
\text { Amostra: } 2895 \text { mulheres em } \\
\text { trabalho de parto. }\end{array}$ & $\begin{array}{l}\text { Por possuir sedação } \\
\text { mínima permitiu que a } \\
\text { equipe de enfermagem } \\
\text { fosse responsável pela } \\
\text { sua administração. }\end{array}$ \\
\hline $\begin{array}{l}\text { RICHARDSON } \\
\text { MG, et al. (2017) }\end{array}$ & EUA & $2 \mathrm{~B}$ & $\begin{array}{l}\text { Tipo de estudo: Estudo de } \\
\text { Coorte. } \\
\text { Amostra: } 6507 \text { mulheres em } \\
\text { trabalho de parto. }\end{array}$ & $\begin{array}{l}\text { Seis em cada } 10 \\
\text { parturientes que } \\
\text { escolheram o óxido } \\
\text { nitroso para o trabalho } \\
\text { de parto demostraram } \\
\text { altos níveis de } \\
\text { satisfação. }\end{array}$ \\
\hline $\begin{array}{l}\text { ROSS JA, et al } \\
\text { (1999) }\end{array}$ & $\begin{array}{l}\text { Reino } \\
\text { Unido }\end{array}$ & $2 \mathrm{~B}$ & $\begin{array}{l}\text { Tipo de estudo: Estudo de } \\
\text { Coorte. } \\
\text { Amostra: } 221 \text { mulheres em } \\
\text { trabalho de parto. }\end{array}$ & $\begin{array}{l}\text { A administração } \\
\text { do } \mathrm{N}_{2} \mathrm{O} \text { foi } \\
\text { considerada uma } \\
\text { técnica segura, não } \\
\text { alterando os escores } \\
\text { de Apgar. }\end{array}$ \\
\hline $\begin{array}{l}\text { STEWART LS e } \\
\text { COLLINS M } \\
(2012)\end{array}$ & EUA & $2 \mathrm{~B}$ & $\begin{array}{l}\text { Tipo de estudo: Estudo de } \\
\text { Coorte. } \\
\text { Amostra: } 200 \text { mulheres em } \\
\text { trabalho de parto. }\end{array}$ & $\begin{array}{l}\text { Equipe de enfermagem } \\
\text { como centro do } \\
\text { cuidado à parturientes } \\
\text { em uso do óxido } \\
\text { nitroso, visto que este } \\
\text { possui sedação } \\
\text { mínima }\end{array}$ \\
\hline
\end{tabular}

Fonte: Dados da pesquisa.

Legenda: NE: Nível de evidência. 


\section{DISCUSSÃO}

Observa-se que as publicações relacionadas ao uso óxido nitroso $\left(\mathrm{N}_{2} \mathrm{O}\right)$ como analgésico intensificaramse no ano de 2012. Não obstante, esse foi o ano em que a Divisão de Instrumentos Porter da Parker Hannifin Corporation garantiu os direitos para fabricar o aparelho Nitronox, o único aparato autorizado pelo Estados Unidos da América (EUA), reintroduzindo o equipamento no mercado, removendo uma grande barreira da disponibilidade de $\mathrm{N}_{2} \mathrm{O}$. Logo, muitas instituições interessadas em fornecer às parturientes a variedade de opções analgésicas começaram a instalar em sua rede hospitalar o uso de óxido nitroso, porém com vários testes da sua eficácia, justificando o grande aumento de publicações a partir deste ano (COLLINS M, et al., 2012; MIGLIACCIO L, et al., 2017).

\section{Aplicação do óxido nitroso como analgésico em parturientes}

O óxido nitroso $\left(\mathrm{N}_{2} \mathrm{O}\right)$ tem sido utilizado em diversos países (Grã-Bretanha, Canadá, Austrália, Finlândia, Estados Unidos) como uma das medidas analgésicas eficazes (ROSS JA, et al., 1999; COLLINS M, et al., 2012; STEWART LS e COLLINS M, 2012; BURGOS J., et al., 2013; MIGLIACCIO L, et al., 2017; RICHARDSON MG, et al., 2017) para trabalho de parto, podendo ser empregado em todos seus estágios (COLLINS M, et al., 2012).

O seu mecanismo de ação é extremamente complexo e não está claramente estabelecido (COLLINS M, et al., 2012), mas a utilização é composta em sua grande maioria com de $50 \%$ de óxido nitroso e $50 \%$ oxigênio, (COLLINS M, et al., 2012; MIGLIACCIO L, et al., 2017, RICHARDSON MG, et al., 2017) onde a concentração de oxigênio não pode ser alterada o que difere daqueles que são utilizados em consultórios odontológicos (CARSTONIU J, et al., 1994; MIGLIACCIO L, et al., 2017).

Além disso, tem início de ação (30 a 50 segundos após a iniciação de inalação) e eliminação rápidas (STEWART LS e COLLINS M, 2012), consequentemente não acumula em tecidos maternos ou fetais, ou seja, não afeta a contratilidade uterina, o bem-estar fetal, os índices de Apgar ou ocasiona depressão respiratória ou a necessidade a de ressuscitação neonatal. (STEWART LS e COLLINS M, 2012; MIGLIACCIO L, et al., 2017; SUTTON CD, et al., 2017)

Outro benefício seria sua sedação mínima (MIGLIACCIO L, et al., 2017; STEWART LS e COLLINS M, 2012), permitindo que as parturientes permaneçam acordadas e alertas, com função motora e sensorial preservadas, sem comprometer o reflexo laríngeo, vias aéreas, a ventilação e a função cardiovascular logo, diminui os riscos de aspiração (COLLINS M, et al., 2012). Quando associado à outras terapias não farmacológicas (imersão em água, psicoprofilaxia e hipnose) pode potencializar o efeito terapêutico (STEWART LS e COLLINS M, 2012).

O óxido nitroso é benéfico não só para controle da analgesia durante o parto, mas também pode ser utilizado em outros procedimentos no pós-parto como: correção de laceração, fórceps, exploração uterina, remoção manual da placenta e curetagem uterina (BISHOP JT, 2007; BERLIT S, et al., 2013; COLLINS M, 2015), assim como pode ser útil para colocação da anestesia neuroaxial (STEWART LS e COLLINS M, 2012; MIGLIACCIO L, et al., 2017).

Embora o óxido nitroso tende a ser menos eficaz do que a analgesia neuroaxial (epidural ou raquiperidural), muitas mulheres que optam pelo seu uso tendem a continuar administrá-lo, apesar da ampla disponibilidade da analgesia neuroaxial além de relatarem alto grau de satisfação após a sua utilização. (COLLINS M, et al., 2012).

Além disso, a analgesia neuroaxial pode ocasionar efeitos adversos como: eventos hipotensivos agudos, desaceleração de contrações uterinas, prolongamento da segunda etapa do trabalho de parto e hiperemia. E historicamente, aumenta o risco para parto cesáreo e além de necessitar de acesso venoso, cateterismo vesical, monitorização fetal contínua (STEWART LS e COLLINS M, 2012). Algumas contraindicações para seu uso seriam: dificuldade física para segurar máscara facial, hábitos etilistas ou uso de droga, pacientes hemodinamicamente instáveis ou com problemas de oxigenação, deficiência da vitamina B12, cirurgia intraocular, obstrução intestinal ou cirurgia do ouvido médio. (STEWART LS e COLLINS M, 2012) 
Os efeitos colaterais mais comumente relatados ocasionados pelo óxido nitroso são náusea, disforia, vertigem, inquietação e ansiedade (COLLINS M, et al., 2012; STEWART LS e COLLINS M, 2012; BURGOS J, et al., 2013; RICHARDSON MG, et al., 2017). Porém, alguns autores (VOLMANEN et al., 2011; STEWART LS e COLLINS M, 2012) relatam que náuseas e vômitos são comuns no trabalho de parto, mesmo sem uso de medicação.

Embora seja de baixo custo, as preocupações com as despesas para sua instalação podem ser uma barreira. Mesmo com tempo, suprimento e recursos humanos menores do que são gastos para colocação e monitoração de uma epidural não se pode esquecer dos resultados satisfatórios no controle da dor (STEWART LS e COLLINS M, 2012; MIGLIACCIO L, et al., 2017).

\section{Assistência de enfermagem a parturientes em uso de oxido nitroso}

Em 2010, o Colégio Americano de Enfermeiras Parteiras publicou uma declaração favorável ao uso do óxido nitroso (AMERICAN COLLEGE NURSE-MIDWIVES, 2010; STEWART LS e COLLINS M, 2012, AMERICAN COLLEGE NURSE-MIDWIVES, 2013) delegando à equipe de enfermagem a coleta de informações, supervisão, avaliação clínica contínua e a administração em parturientes, visto que este possui sedação mínima (KIN TL, 2011; STEWART LS e COLLINS M, 2012; MIGLIACCIO L, et al., 2017).

Enfermeiros precisam estar atentos às necessidades e desejos das parturientes no modo de parir, podendo escolher agachar, sentar, usar bolas de parto, chuveiro ou banheira, visto que esta é protagonista do seu parto, exigindo uma supervisão e avaliação precisa da vitalidade fetal e bem-estar materno (MIGLIACCIO L, et al., 2017).

Possui papel educador pois deve ensinar a parturiente a técnica correta, esclarecer o efeitos colaterais, visando a segurança e satisfação da paciente, maximizando dos resultados (COLLINS M, et al., 2012; STEWART LS e COLLINS M, 2012; MIGLIACCIO L, et al., 2017) bem como o controle dos sinais vitais, de riscos e estágios do parto e o bem-estar materno ou fetal. Como qualquer modo de analgesia ou anestesia ao parto é necessário também o consentimento da paciente ou familiar responsável. Logo, a enfermeira será fundamental na obtenção dessa assinatura, detalhando riscos e benefícios do seu uso (STEWART LS e COLLINS M, 2012). Deve documentar o tempo de inicio da ação, termino, efeitos colaterais ou complicações e eficácia da analgesia (MIGLIACCIO L, et al., 2017).

\section{CONSIDERAÇÕES FINAIS}

A utilização do oxido nitroso é uma alternativa segura para o alívio da dor em parturientes. A utilização desse método exige extensa colaboração entre os profissionais e múltiplos departamentos e comitês hospitalares, em especial da equipe de enfermagem, sendo de sua competência a supervisão e avaliação clínica da paciente. Há evidentes desafios para sua utilização, porém inúmeras vantagens, como o seu baixo custo. $\mathrm{O}$ acesso à essa terapia ainda é limitada e pouco difundida, evidenciando a necessidade de ampliar pesquisar e estudos a respeito dessa modalidade terapêutica. Logo, esse estudo pretende estimular e subsidiar novas pesquisas que trabalhem e aprofundem o uso do oxido nitroso além de outros métodos farmacológicos e não farmacológicos que possam ser aplicados para o alivio da dor durante o parto, tornando-o mais humanizado e menos temeroso para algumas mulheres.

\section{REFERÊNCIAS}

1. AMERICAN COLLEGE OF NURSE-MIDWIVES. Nitrous oxide for labor analgesia. Rev. Journal of idwifery \& Women's Healt. 2010,55(2): 292-296. doi:10.1016/j.jmwh.2010.03.003.

2. AMERICAN COLLEGE OF NURSE MIDWIVES. Nitrous oxide for pain relief in labor. Share with Women. $J$ Midwifery Womens Health. 2013;58(6):727-728.

3. BARBIERI R, et al. Nitrous oxide for labor pain. Rev. OBG Manag. 2014;26(12):10-12.

4. BERLIT S, et al. Effectiveness of nitrous oxide for postpartumperineal repair: a randomised controlled trial. Rev. Eur J Obstet Gynecol Reprod Biol. 2013;170(2):329-332. 
5. BISHOP JT. Administration of nitrous oxide in labor: expanding the options for women. J Midwifery Womens Health. 2007;52:308-309.

6. BOBB LE, et al. Does nitrous oxide labor analgesia influence the pattern of neuraxial analgesia usage? An impact study at an academic medical center. Rev. J Clin Anesth. 2016;(35):54-57.

7. BURGOS J, et al. Nitrous oxide for analgesia in external cephalicversion at term: prospective comparative study. Rev. J. Perinat. Med. 2013;41(6):719-723. doi: 10.1515/jpm-2013-0046.

8. CARSTONIU $J$, et al. Nitrous oxide in early labor. Safety and analgesic efficacy assessed by a doubleblind, placebo-controlled study. Rev. Anesthesiology. 1994;80(1):30-5.

9. COLLINS M, et al. Nitrous Oxide for Labor Analgesia: Expanding Analgesic Options for Women in the United States. Rev Obstet Gynecol. 2012;5(3-4):126-131.

10. COLLINS M. A case report on the anxiolytic properties of nitrous oxide during labor. Rev. J Obstet Gynecol Neonatal Nurs. 2015;44(1), 87.

11. DURIEUX N, t al. Médecine Factuelle: la hiérarchisation des preuves par le Centre for Evidence-Based Medicine d'Oxford. Rev Med Liège. 2013;68(12):644-649.

12. KIN TL. From forgotten to mainstream: How a nursemidwife's commitment to nitrous oxide changed practice. Rev. Journal of Midwifery \& Women's Health. 2011,56(6):541-542. doi:10.1111/j.1542-2011.2011.00131.x.

13. LOCKWOOD C, et al. Chapter 2: Systematic reviews of qualitative evidence. In: Aromataris E, Munn Z (Editors). Joanna Briggs Institute Reviewer's Manual. The Joanna Briggs Institute. 2017.

14. MENDES KDS, et al. Revisão integrativa: método de pesquisa para a incorporação de evidências na saúde e na enfermagem. Rev. Texto \& Contexto Enfermagem. Florianópolis. 2008;17(4):758-64.

15. MIGLIACCIO L, et al. Initiating Intrapartum Nitrous Oxide in an AcademicHospital: Considerations and Challenges. Rev. Journal of Midwifery \&Women's Health. 2017;62(3):358-362. doi: 10.1111/jmwh.12635.

16. RICHARDSON MG, et al. Nitrous Oxide During Labor: Maternal Satisfaction Does Not Depend Exclusively on Analgesic Effectiveness. Rev. Anesth Analg. 2017;124(2):548-553. doi: 10.1213/ANE.0000000000001680.

17. ROSS JA, et al. The use of $0.25 \%$ isoflurane premixed in $50 \%$ nitrous oxide and oxygen for pain relief in labour. Rev. Anaesthesia. 1999;54(12):1166-1172.

18. STEWART LS, COLLINS M. Nitrous oxide as labor analgesia: clinical implications for nurses. Rev. Nurs Womens Health. 2012;16(5):398-408. doi: 10.1111/j.1751-486X.2012.01763.x.

19. SUTTON CD, et al. Nitrous oxide for labor analgesia: Utilization and predictors of conversion to neuraxial analgesia. Rev. J Clin Anesth. 2017;40(2): 40-45. doi: 10.1016/j.jclinane.2017.04.005.

20. Volmanen $P$, et al. Alternatives to neuraxial analgesia for labor. Current Opinion in Anesthesiology, 2011, 24(3), 235-241. doi:210.1097/ACO.1090b1013e328345ad328318. 